\title{
Does transperitoneal minimally invasive radical prostatectomy increase the amount of small bowel receiving salvage radiation?
}

\author{
Murilo A. Luz, MD; ${ }^{*}$ Alan Dal Pra, MD; ${ }^{*}+$ Hin-Yu Vincent Tu, MD; ${ }^{*}$ Marie Duclos, MD; ${ }^{*+}$ Fabio L.B. Cury, MD; ${ }^{*+}$ \\ Bassel G. Bachir, MD,* Armen G. Aprikian, MD, FRCSC,; Simon Tanguay, MD, FRCSC,; \\ Wassim Kassouf, MD, FRCSC*
}

*Department of Surgery, Division of Urology, McGill University, Montreal, QC; 'Department of Radiation Oncology, McGill University, Montreal, QC

See related article on page 449.

Cite as: Can Urol Assoc J 2013;7(11-12):444-8. http://dx.doi.org/10.5489/cuaj.265 Published online December 5, 2013.

\section{Abstract}

Introduction: Transperitoneal minimally invasive radical prostatectomy (MIRP) has become first choice for several urologists and patients dealing with localized prostate cancer. We evaluate the effect of postoperative radiation on the small bowel in patients who underwent extraperitoneal open versus transperitoneal MIRP. Methods: We reviewed all patients who received postoperative radiation from 2006 to 2010 . Planning target volume (PTV) and surrounding organs, including the small bowel, were delineated. The presence of the small bowel in PTV and its volume in receiving each dose level were analyzed.

Results: A total of 122 patients were included: 26 underwent MIRP and 96 underwent open prostatectomy. The median age of patients was 66 years, with median body mass index $27 \mathrm{~kg} / \mathrm{m}^{2}$. The total PTV dose was $66 \mathrm{~Gy}$, with the minimum and maximum doses received by the small bowel 0.4 and $66.4 \mathrm{~Gy}$, respectively. The maximum volume of small bowel that received the safe limit of $40 \mathrm{~Gy}$ was $569 \mathrm{~cm}^{3}$. Of the 26 patients who underwent MIRP, $12(46 \%)$ had small bowel identified inside the PTV compared to 57 (59\%) among patients who underwent open prostatectomy $(p=0.228)$. The mean volume of the small bowel receiving 40 Gy was 26 and $67 \mathrm{~cm}^{3}$ in open and MIRP groups, respectively $(p=0.006)$; the incidence of acute complications was the same in both groups.

Conclusions: Higher volumes of the small bowel are subjected to significant radiation after MIRP procedures compared to open procedures; however, we could not demonstrate any impact on acute complications. Whether there is a difference in late complications remains to be evaluated.

\section{Introduction}

Prostate cancer is the most prevalent cancer and the second cause of cancer-related mortality in the United States. ${ }^{?}$
Radical local management with surgical extirpation or radiation remains a common treatment for newly diagnosed patients. ${ }^{2}$ Minimally invasive radical prostatectomy (MIRP) is a first choice for several urologists and patients dealing with localized prostate cancer. Although the benefits of minimally invasive approaches for prostate cancer are still debatable, in 2011 robotic prostatectomies represented more than $80 \%$ of all radical prostatectomies performed throughout the United States. ${ }^{3}$ Even though an extraperitoneal access is feasible when performing this type of surgery, the most common approach has become the transperitoneal access.

Several risk factors found at prostatectomy specimen, including stage, positive margins and seminal vesicle involvement, will put patients at risk of local recurrence. ${ }^{4}$ In light of current data, we should consider adjuvant radiation for all patients with high-risk features at prostatectomy specimens. ${ }^{5}$ Even patients with few or no risk factors at initial assessment may eventually develop local recurrence.

When facing biochemical failure with no evidence of distant metastasis, most patients will be offered salvage radiation therapy. ${ }^{6}$ When an intraperitoneal approach is used during MIRP, the bladder detachment from the anterior abdominal wall could precipitate small bowel adhesions close to the prostatic bed, ultimately in the area of future salvage irradiation. We evaluate the effect of salvage radiation on the small bowel in patients who underwent extraperitoneal open versus transperitoneal MIRP.

\section{Methods}

This study conforms to the ethical guidelines of the Helsinki declaration. We included patients treated with radiation following transperitoneal MIRP or extraperitoneal open prostatectomy from 2006 to 2010 . All of them were subjected to salvage radiation to the prostatic bed using a $3 \mathrm{D}$ conformal technique. The total dose of salvage radiation was 66 Gy. The planning target volume (PTV) and surrounding organs, including the small bowel, were delineated. We contoured 
all small bowel loops at least 10 slices above the PTV. As per definition, the PTV receives the complete dose (66 Gy) in at least $95 \%$ of its volume. In both groups, the bladder was kept full with a comfortable volume throughout the treatment to minimize bladder and small bowel exposure to radiation. Patients had a median follow-up of 12 months after radiation treatment. Initially, we analyzed the presence of any small-bowel volume in the PTV. Then, we analyzed the small-bowel volume for each dose level $(10,20,30$ or $40 \mathrm{~Gy}$ ), along with minimal and maximal amount of radiation applied. A dose of $<40$ Gy was the acceptable limit on the small bowel. ${ }^{7}$ Additionally, up to $150 \mathrm{~cm}^{3}$ of small bowel is considered by radiation oncologists as a safe volume to receive radiation. We used this small-bowel volume and again compared the amount of radiation in both groups. Finally, acute toxicity related to radiation treatment was analyzed. The toxicity criteria used was the already-validated Common Toxicity Criteria (CTC) version 3.0 scoring system. ${ }^{8}$

\section{Results}

A total of 122 patients were included; 26 underwent transperitoneal MIRP and 96 patients had an extraperitoneal open prostatectomy. No patient had a history of inflammatory bowel disease or previous pelvic surgery. All patients completed radiation treatment with 66 Gy of total dose delivered in 33 daily fractions. The median age was 66 years (interquartile range [IQR], 59-73) and median body mass index (BMI) was $27 \mathrm{~kg} / \mathrm{m}^{2}$ (IQR 18-36). The maximum and minimum dose received by any amount of small bowel was 66.4 and 0.4 Gy, respectively (Fig. 1).

Both groups were comparable with regards to BMI and PTV (Table 1). Patients who underwent open prostatectomies were 4 years older on average and had slightly bigger bladder volumes on computed tomography (CT).

When the presence or absence of the small bowel inside the PTV was evaluated (which means some volume of small bowel is inside the main area of treatment), there was no statistically significant difference between the 2 groups; $59 \%$ and $46 \%$ of patients had small bowel inside the PTV area in open and MIRP groups, respectively $(p=0.228)$ (Figure 2, part A).

In total, $75 \%$ of patients received at least 40 Gy of radiation on some volume of the small bowel. The maximum small-bowel volume receiving 40 Gy was $569 \mathrm{~cm}^{3}$. The mean small-bowel volume receiving 40 Gy was 26 and $67 \mathrm{~cm}^{3}$ for patients who underwent open and MIRP, respectively ( $p=0.006$ ) (Fig. 2, part B). Overall, most patients $(91 \%)$ received doses less than 30 Gy on $150 \mathrm{~cm}^{3}$ of the small bowel. Comparing groups, $2 \%$ and $15 \%$ of patients received more than $40 \mathrm{~Gy}$ on $150 \mathrm{~cm}^{3}$ of the small bowel in open and MIRP groups, respectively $(p=0.005)$ (Fig. 2 , part C). After adjusting for age, BMI, bladder volume and performance of pelvic lymphadenectomy, patients treated with MIRP remained at a significantly increased risk of receiving at least 40 Gy of radiation to $150 \mathrm{~cm}^{3}$ or more of their small bowel compared to those treated with open prostatectomy (hazard ratio 1.28, $p=0.02$ ). Acute small bowel toxicity did not differ between the 2 groups. Acute toxicity grade I or II was present in $40 \%$ and $46 \%$ of patients submitted to open or MIRP, respectively.

\section{Discussion}

In this study, we demonstrated that following MIRP, patients will receive extra doses of salvage radiation to a larger
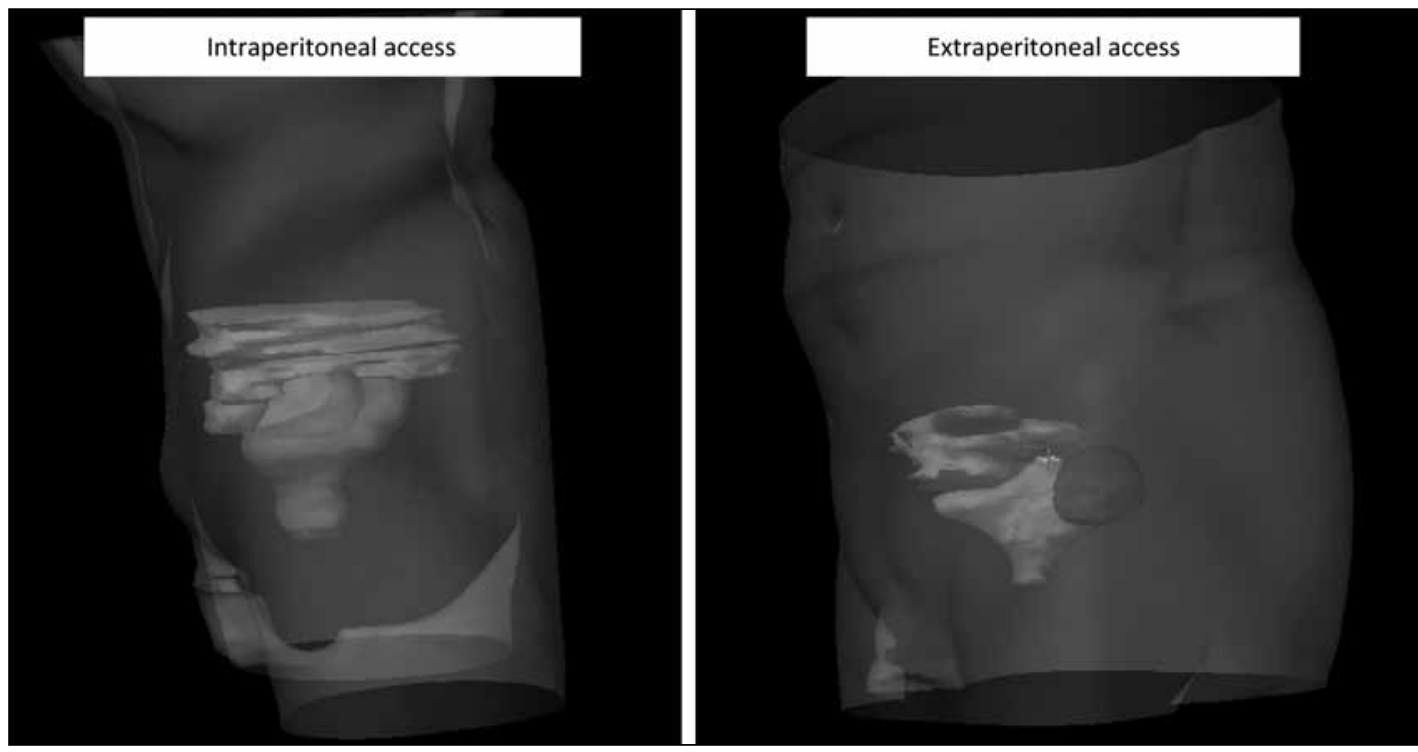

Fig. 1. Differences in radiation treatment planning between extraperitoneal and intraperitoneal access at the moment of radical prostatectomy. Small bowel (brown) insinuates lower in the pelvis after intraperitoneal access. Red corresponds to planning target volume, blue (intraperitoneal) and yellow (extraperitoneal) corresponds to bladder volume. 
Luz et al.

\begin{tabular}{|c|c|c|c|}
\hline Surgery & MIRP & Open & $p$ value \\
\hline Age (median, years) & 62 & 66 & 0.021 \\
\hline BMI (median, kg/m²) & 26.8 & 27.1 & 0.890 \\
\hline Bladder volume (mean, $\mathrm{cm}^{3}$ ) & 135.9 & 196.3 & 0.041 \\
\hline PTV (mean, $\mathrm{cm}^{3}$ ) & 350.3 & 392.8 & 0.241 \\
\hline
\end{tabular}

amount of the small bowel compared to patients who underwent conventional extraperitoneal open prostatectomies.

With new techniques and new surgical procedures, surgeons and patients face situations and outcomes that they never thought of before. For many years, the classical retropubic radical prostatectomy, first described by Walsh in $1980,{ }^{9}$ was the gold standard in the surgical treatment of localized prostate cancer. Since the evolution of laparoscopic techniques and advanced robotic assisted surgery, surgeons are now dealing with new issues regarding surgical care decisions. Initial descriptions towards minimally invasive surgical treatment of prostate cancer were made by Schuessler and colleagues almost 20 years ago. ${ }^{10}$ However, the first larger series of laparoscopic radical prostatectomy was published by Guillonneau and colleagues in 1999. ${ }^{11}$ An extraperitoneal approach is feasible and demonstrated good results as described initially by Raboy and colleagues in 1997; however, most surgeons performing minimally invasive prostatectomy, including those at our centre, are using the intraperitoneal approach.

Long-term effects of radiation are well-described. With time, it has become less aggressive; new techniques are more targeted to specific tumours. However, a perfect match between effective treatment and no scattered dose remains a challenge. Healthy organs are still receiving variable doses of radiation, along with its associated consequences. ${ }^{12,13}$ Much is known about the late intestinal side effects of postoperative radiation, including diarrhea, constipation, adhesions, strictures and perforations. On the other hand, little has been published about its acute complications. A recent study reports a case of acute enteritis causing small bowel obstruction; this highlights the importance of using caution when planning radiation treatment close to the small bowel. ${ }^{14}$

Surgeons and radiation oncologists know the risks of severe complications related to small bowel exposure to radiation, a diagnosis well-defined as actinic enteritis. Any patient receiving radiation to the pelvis has a risk for this kind of complication. ${ }^{15}$ In this study, we demonstrated that after a transperitoneal minimally invasive prostatectomy, patients will receive extra doses to a larger amount of the small bowel compared to patients who underwent conventional open prostatectomies. There is no effective way to displace the small bowel from the pelvis, although a full bladder could help and even a tissue expander was already tested to prevent small bowel from receiving excessive doses of radiation. ${ }^{16}$

In addition, others have shown that patient positioning, particularly the Trendelenburg position, may reduce the volume of the small bowel in the PTV. ${ }^{17,18}$ It is possible that transection of the umbilical ligaments and urachus during transperitoneal MIRP and detachment of the bladder from the anterior abdominal wall resulted in a higher small-bowel volume around the prostatic bed and PTV. Therefore, this may provide a rationale for keeping the bladder attached to the umbilicus anteriorly during the transperitoneal approach, thereby keeping the bowel higher up and further away from the PTV. On the other hand, an argument can be made for resuturing the bladder to the anterior abdominal wall, especially in high-risk patients who may require adjuvant or salvage radiotherapy.

Finelli and colleagues have previously compared simulated radiation fields for patients who underwent postoperative radiation regarding small bowel toxicity. ${ }^{19}$ They concluded that transperitoneal laparoscopic prostatectomy did not increase the presence of the small bowel within the target volume for postoperative radiotherapy. That study compared hypothetical cases and only considered the presence or not of small bowel inside the main radiation field (PTV). We initially performed the same analysis and have also found no significant difference between the 2 groups for this specific criterion. However, it is imperative to note that this single variable considers even tiny amounts of small bowel receiving very small doses of radiation as positive, which definitively does not correlate with clinical consequences. After performing a more detailed analysis, the conclusions from our study did not concur with those of Finelli and colleagues. In our study, we exclusively selected cases that effectively had adjuvant or salvage radiation. We also analyzed multiple parameters, including the amount of small bowel receiving each level of dose as well as minimal and maximum dose. This is more reflective of the radiation effect on the small bowel; we were, therefore, able to conclude that larger volumes of the small bowel receive significant doses of radiation when transperitoneal approaches are used.

We do acknowledge some limitations of our study. First, although it can be a challenge to mark on a CT scan the space occupied by the small bowel; we did nevertheless apply a standardized way to accomplish this. Second, to reflect current practice, we did not analyze the effect of expanding the area of radiation to the pelvic fields (lymph nodes), in addition to prostatic bed as the clinical benefit of this approach remains to be demonstrated. We hypothesize that even more parts of the small bowel will receive radiation in the MIRP group once the field is expanded to the pelvic lymph nodes. Third, we did not take into account possible daily shifts in bowel contents and assumed that the bowel location is more or less constant. However, taking 


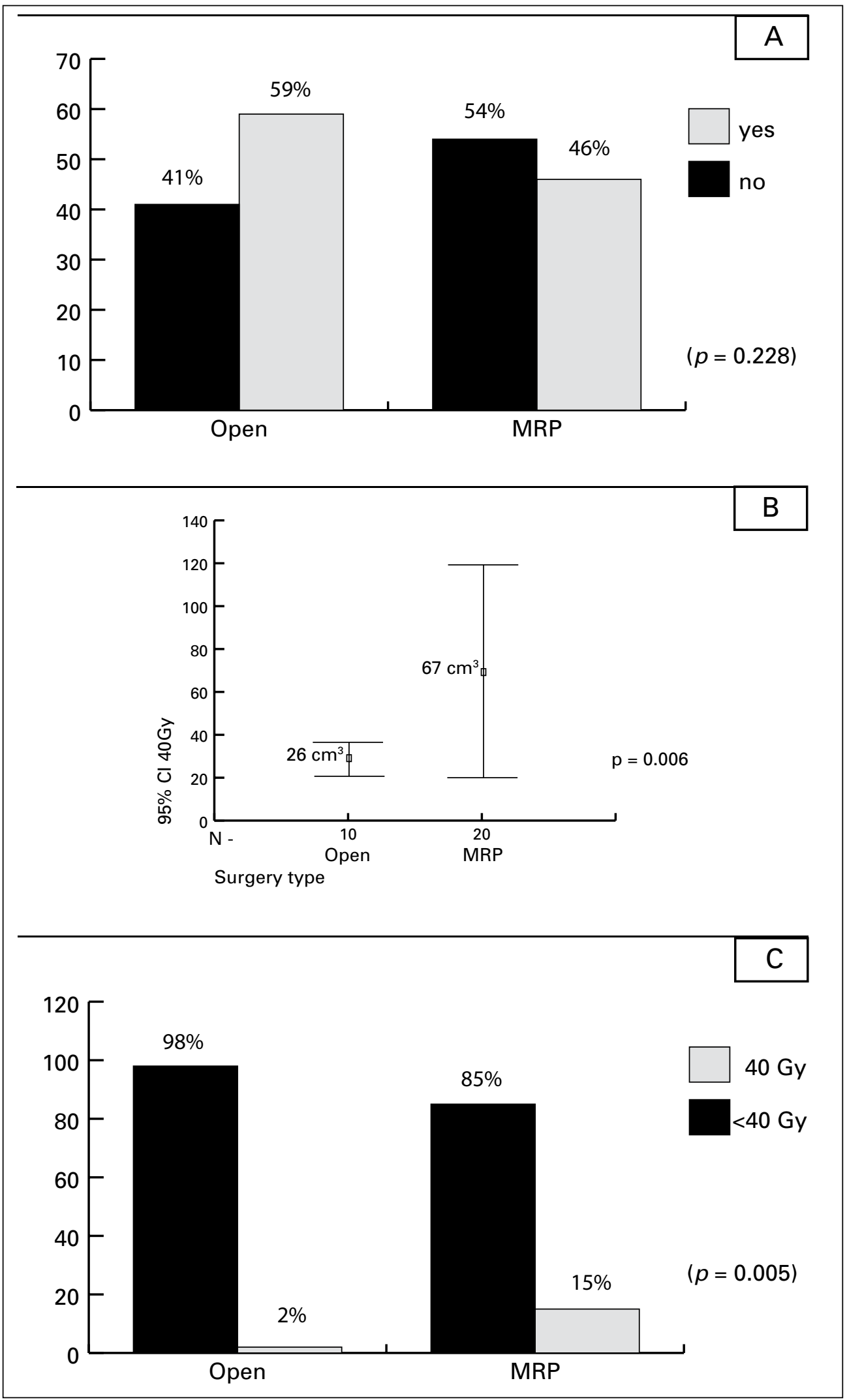

Fig. 2. (A) presence of any volume of small bowel inside the planning target volume $(\%)(p=0.228)$, (B) mean volume of small bowel receiving $40 \mathrm{~Gy}$ of radiation, (C) percentage of patients that received $40 \mathrm{~Gy}$ or more radiation dose to at least $150 \mathrm{~cm}^{3}$ of small bowel $(p=0.005)$. 
Luz et al.

into consideration surgical adhesions, it is often that small bowel loops will keep their positions. Lastly, our study is limited by its retrospective nature, inter-surgeon variability in surgical technique, and small patient cohort, particularly in the MIRP group.

\section{Conclusions}

Higher volumes of the small bowel are submitted to radiation after transperitoneal MIRP procedures comparing to extraperitoneal open procedures. However, we found no impact on the incidence of acute complications. Whether there is a difference in late complications remains to be evaluated.

Competing interests: Dr. Aprikian is a board observer for Bioniche Inc., a member of the Speakers bureau for Amgen, Abbvie and Astellas. He has also received grants from Amgen, Astellas and Abbvie. Moreover, he has participated in a clinical trial within the past 2 years for Astellas. Dr. Kassouf is an Advisory Board member and a speaker for Amgen and Astellas. He has also received grants and honoraria from these companies. He is currently participating in unpaid clinical trials within the past 2 years. Dr. Luz, Dr. Dal Pra, Dr. Tu, Dr. Duclos, Dr. Cury, Dr. Bachir and Dr. Tanguay all declare no competing financial or personal interests.

This paper has been peer-reviewed.

\section{References}

1. Jemal A, Segal R, XU J, et al. Cancer Statistics, 2010. CA Cancer J Clin 2010;60:277-300. http:// dx.doi.org/10.3322/caac.20073

2. Mohan R, Schellhammer PF. Treatment options for localized prostate cancer. Am Fam Physician 2011;84:413-20.

3. Bianco, F. Robotic radical prostatectomy: Present and future. Arch Esp Urol 2011;64:839-46.

4. Raboy A, Ferzli G, Albert P. Initial experience with extraperitoneal endoscopic radical retropubic prostatectomy. Urology 1997;50:849-53. http://dx.doi.org/10.1016/S0090-4295(97)00485-8

5. Thompson IM, Tangen CM, Paradelo J, et al. Adjuvant radiotherapy for pathological T3NOMO prostate cancer significantly reduces risk of metastases and improves survival: long-term follow-up of a randomized clinical trial. J Urol 2009;181:956-62. http://dx.doi.org/10.1016/i.juro.2008.11.032
6. Stephenson AJ, Bolla M, Briganti A, et al. Postoperative radiation therapy for pathologically advanced prostate cancer after radical prostatectomy. Eur Urol 2012;61:443-51. http://dx.doi.org/10.1016/i. eururo.2011.10.010

7. Tho $L M$, Glegg M, Paterson J, et al. Acute small bowel toxicity and preoperative chemoradiotherapy for rectal cancer: investigating dose-volume relationships and role for inverse planning. Int J Radiat Oncol Biol Phys 2006;66:505-13 Epub 2006 Jul 31.

8. Nath SK, Sandhu AP, Rose BS, et al. Toxicity analysis of postoperative image-guided intensity-modulated radiotherapy for prostate cancer. Int I Radiat Oncol Biol Phys 2010;78:435-41. http://dx.doi. org/10.1016/i.i.irobp.2009.08.023

9. Walsh PC, Jewett HJ. Radical surgery for prostatic cancer. Cancer 1980;45:1906-11.

10. Schuessler WW, Schulam PG, Clayman RV, et al. Laparoscopic radical prostatectomy: initial short-term experience. Urology 1997;50:854-7. http://dx.doi.org/10.1016/S0090-4295(97)00543-8

11. Guillonneau B, Cathelineau X, Barret E, et al. Laparoscopic radical prostatectomy: technical and early oncological assessment of 40 operations. Eur Urol 1999;36:14-20. http://dx.doi.org/10.1159/000019921

12. Bekelman JE, Mitra N, Efstathiou J, et al. Outcomes after intensity-modulated versus conformal radiotherapy in older men with nonmetastatic prostate cancer. Int J Radiat Oncol Biol Phys 2011;81:e325-34. http:// dx.doi.org/10.1016/i.ijrobp.2011.02.006

13. Nakamura N, Shikama N, Takahashi 0 , et al. The relationship between the bladder volume and optimal treatment planning in definitive radiotherapy for localized prostate cancer. Acta Oncol 2012;51:730-4. http://dx.doi.org/10.3109/0284186X.2011.639388

14. Curtis NJ, Bryant T, Raj S, et al. Acute radiation enteritis causing small bowel obstruction. Ann R Coll Surg Engl 2011;93:e129-30. http://dx.doi.org/10.1308/10.1308/147870811X602122

15. Dearnaley D, Syndikus I, Sumo G, et al. Conventional versus hypofractionated high-dose intensity-modulated radiotherapy for prostate cancer: preliminary safety results from the CHHiP randomized controlled trial. Lancet Oncol 2012;13:43-54. http://dx.doi.org/10.1016/S1470-2045(11)70293-5

16. McKay GD, Wong K, Kozman DR. Laparoscopic insertion of pelvic tissue expander to prevent radiation enteritis prior to radiotherapy for prostate cancer. Radiat Oncol 201 1;6:47. http://dx.doi.org/10.1186/1748717X-6-47

17. Dirier A, Adli M, Andic F, et al. Reduction of irradiated small bowel volume with Trendelenburg position in gynecologic pelvic radiotherapy. J Med Imaging Radiat Oncol 2010;54:142-5. http://dx.doi. org/10.1111/j.1754-9485.2010.02153.x

18. Czigner $\mathrm{K}$, Agoston $P$, Forgács $\mathrm{G}$, et al. Patient positioning variations to reduce dose to normal tissues during 3D conformal radiotherapy for high-risk prostate cancer. Strahlenther Onkol 2012;188:816-22.

19. Finelli $A$, Punnen $S$, Rosewall $T$, et al. Transperitoneal laparoscopic prostatectomy does not increase small bowel within the target volume for postoperative radiotherapy. J Urol 2009; 182:2280-4. http://dx.doi. org/10.1016/i.juro.2009.07.037

Correspondence: Dr. Wassim Kassouf, Division of Urology, McGill University Health Centre, 1650 Cedar Ave., Rm L8-315, Montreal, QC H3G 1A4; fax: 514-934-8297; wassim.kassou@@muhc.mcgill.ca 